\title{
Analysis of a DKA protocol: Laboratory tests and outcomes
}

\author{
Arunee Motes MD, Fabiana Fernandez MD, Hawa Edriss MD
}

\begin{abstract}
Most hospitals use protocols to manage diabetic ketoacidosis using laboratory criteria to monitor the response to insulin and fluid therapy. This study analyzed information collected prospectively on a group of patients with diabetic ketoacidosis to review outcomes and management details. The study included 37 patients with a mean age of $38.1 \pm 18.5$ years. The initial blood glucose was $546.4 \pm 296.3 \mathrm{mg} / \mathrm{dL}$. The initial anion gap was $31.8 \pm 7.8 \mathrm{mEq} / \mathrm{L}$. The mean time to anion gap closure twice was $19.2 \pm 12.8$ hours. The mean fluid administered until anion gap closure was $3694.9 \pm 2484.6 \mathrm{~mL}$. The insulin dose during the first day of management was $69.5 \pm 49.9$ units; the mean number of point-of-care glucose levels during the first 24 hours was $21.2 \pm 6$. The number of basic metabolic panels collected during hospital management was $12.2 \pm$ 13.7. The mean ICU length of stay was $2.5 \pm 3.8$ days with no mortality. This study indicates that patients with diabetic ketoacidosis had a large number of basic metabolic panel tests and a large number of point of care glucose measurements using this protocol. This protocol needs review to determine whether or not the number of tests can be reduced, and transfer out of the ICU can occur more quickly.
\end{abstract}

Keywords: Diabetic ketoacidosis, glucose levels, anion gap, basic metabolic panels, outcomes

\section{INTRODUCTION}

Most hospitals use standardized insulin and fluid protocols for managing patients with diabetic ketoacidosis (DKA). These protocols initiate a series of steps, including laboratory tests, fluid administration, insulin administration, and electrolyte correction. Most patients recover and, if initially admitted to the ICU, move out of the ICU or are discharged from the hospital. The physicians and nurses involved in the care of these patients usually judge overall management results by the main outcome, namely recovery and discharge, and do not review or analyze the details of the protocol. Physicians working in the medical intensive care unit at University Medical Center (UMC), Lubbock, Texas, recently completed a prospective

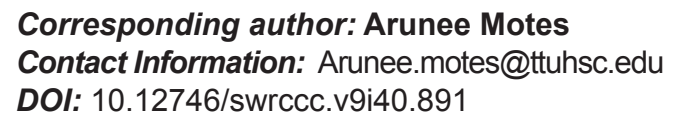

study on the formation of advanced glycation end products in patients with DKA; the information collected during that study provides an opportunity to analyze the timelines for the improvement in the metabolic disorder and the laboratory tests needed to manage the protocol.

\section{DEFINITION}

Diabetic ketoacidosis is a life-threatening complication in patients with diabetes mellitus that has significant morbidity and mortality; it usually occurs in patients with type 1 diabetes but can also occur in patients with type 2 diabetes. Diabetic ketoacidosis is a medical emergency that should be diagnosed and managed promptly.

Diabetic ketoacidosis is characterized by the triad of hyperglycemia, metabolic acidosis, and ketosis. The diagnosis requires hyperglycemia $(>250 \mathrm{mg} / \mathrm{dL})$, metabolic acidosis $(\mathrm{pH}<7.30)$, a bicarbonate level less than or equal to $18 \mathrm{mEq} / \mathrm{L}$, and increased ketone 
Table 1. Diagnostic Criteria for Diabetic Ketoacidosis Severity

\begin{tabular}{|l|l|l|l|}
\hline Criterion & Mild DKA & Moderate DKA & Severe DKA \\
\hline Anion gap & $>10 \mathrm{mEq} / \mathrm{L}$ & $>12 \mathrm{mEq} / \mathrm{L}$ & $>12 \mathrm{mEq} / \mathrm{L}$ \\
\hline Arterial $\mathrm{pH}$ & $7.24-7.30$ & $7.00-<7.24$ & $<7.00$ \\
\hline Serum osmolality & Variable & Variable & Variable \\
\hline Mental status & Alert & Alert/drowsy & Stupor/coma \\
\hline Serum bicarbonate & $15-18 \mathrm{mEq} / \mathrm{L}$ & $10-<15 \mathrm{mEq} / \mathrm{L}$ & $<10 \mathrm{mEq} / \mathrm{L}$ \\
\hline
\end{tabular}

Adapted from Diabetes Care 2009 Jul; 32(7):1335-1343.

concentration. Beta-hydroxybutyrate is a better measurement of the degree of ketosis, since its levels correlate better with changes in arterial $\mathrm{pH}$ and blood bicarbonate levels than ketone levels do.

The severity of DKA is determined by arterial $\mathrm{pH}$ at the time of diagnosis (Table 1).

\section{Prevalence and incidence of DM/DKA, STATISTIC FOR MORBIDITY, AND MORTALITY}

The National Diabetes Statistics Report 2020 estimated that for the year 201834.1 million adults aged 18 years or older or $13.0 \%$ of all US adults had diabetes. In that year, 1.5 million new cases of diabetes or 6.9 per 1,000 persons were diagnosed. In 2016, a total of 16 million emergency department visits were reported with diabetes as any listed diagnosis among adults aged 18 years or older, including 224,000 for hyperglycemic crisis (9.7 per 1,000 adults with diabetes) and 235,000 for hypoglycemia (10.2 per 1,000 adults with diabetes); the number and rate of hospitalizations were 209,000 for hyperglycemic crisis (9.1 per 1,000 adults with diabetes), including 188,000 for DKA, 21,000 for hyperglycemic hyperosmolar syndrome (HHS) and 57,000 for hypoglycemia (2.5 per 1,000 adults with diabetes). ${ }^{1}$

In 2017, diabetes was the seventh leading cause of death in the United States. This finding is based on 83,564 death certificates in which diabetes was listed as the underlying cause of death (approximate rate, 25.7 per 100,000 persons). In 2017, there were 270,702 death certificates with diabetes listed as the underlying or contributing cause of death (approximate rate 83.1 per 100,000 persons). In adults with DKA, the overall mortality is $<1 \%$; however, a mortality rate $>5 \%$ has been reported in the elderly and in patients with concomitant life-threatening illnesses. ${ }^{1}$

The total direct and indirect estimated cost of diagnosed diabetes in the United States in 2017 was $\$ 327$ billion. Total direct estimated costs of diagnosed diabetes increased from $\$ 188$ billion in 2012 to $\$ 237$ billion in 2017 (2017 dollars); total indirect costs increased from $\$ 73$ billion to $\$ 90$ billion in the same period (2017 dollars). Between 2012 and 2017, excess medical costs per person associated with diabetes increased from $\$ 8,417$ to $\$ 9,601$. $^{1}$

\section{RISK FACTORS, TRIGGERS, AND POPULATIONS}

Most patients with DKA have autoimmune type 1 diabetes; however, patients with type 2 diabetes are also at risk during the catabolic stress of acute illness, such as trauma, surgery, or infections. Other risk factors for DKA include treatment non-compliance and alcohol abuse. ${ }^{2}$ Myocardial infarction is one precipitating cause of DKA that is especially important to consider in older patients with diabetes.

\section{RELATED COMPLICATIONS}

Common complications of DKA include hypoglycemia, hypokalemia, and recurrent hyperglycemia. These complications may be minimized by careful monitoring. Hyperchloremia is also common, but it is a transient finding that usually requires no specific treatment.

Cerebral edema is a rare but important complication of DKA and is more common in young patients. It occurs in 0.5 to 1 percent of all DKA cases with a 
mortality rate of 21 to 24 percent. ${ }^{3}$ The osmolar gradient caused by the high blood glucose results in water shifting from the intracellular fluid to the extracellular fluid space and contraction of cell volume. Correction with insulin and intravenous fluids can result in a rapid reduction in effective osmolarity, reversal of the fluid shift and the development of cerebral edema. ${ }^{4}$ Early signs of cerebral edema include headache, confusion, and lethargy. Papilledema, hypertension, hyperpyrexia, and diabetes insipidus also may occur. Patients typically improve mentally with initial treatment of DKA but then suddenly worsen. Dilated ventricles may be found on computerized tomography or magnetic resonance imaging. If cerebral edema is suspected, the treatment should be initiated immediately and not be delayed for the completion of these studies. Seizures, pupillary changes, and respiratory arrest with brainstem herniation may occur in more severe cases. Once severe presentations occur, the mortality rate is greater than 70 percent, and only about 10 percent of patients recover without any sequelae.

To reduce the occurrence of cerebral edema, it is necessary to avoid overhydration and to limit the rate at which the blood glucose level decreases. Mannitol therapy $(0.25-1 \mathrm{~g} / \mathrm{kg})$ and hyperventilation have been recommended for DKA-related cerebral edema, based on limited evidence.

\section{REVIEW DKA MANAGEMENT}

In 2016, Tran et al. reviewed existing studies investigating inpatient DKA management in adults, focusing on intravenous (IV) fluids, insulin administration, potassium, bicarbonate, and phosphate replacement, and DKA management protocols and impact of DKA resolution rates on outcomes. The review included 85 articles published between 1973 and 2016 and found that there are major deficiencies in evidence for optimal management of DKA. Current practice is guided by weak evidence and consensus opinion. All aspects of DKA management require randomized controlled trials to affirm or redirect management and formulate consensus evidence-based practice to improve patient outcomes. ${ }^{5}$

According to American Diabetes Association (ADA) recommendations regarding IV fluid administration in DKA patients, in the absence of cardiac compromise, isotonic saline $(0.9 \% \mathrm{NaCl})$ is infused at a rate of $15-20 \mathrm{~mL} / \mathrm{kg} / \mathrm{h}$ or $1-1.5 \mathrm{~L}$ during the first hour. The subsequent choice for fluid replacement depends on hemodynamics, the state of hydration, serum electrolyte levels, and urinary output. In general, $0.45 \% \mathrm{NaCl}$ infused at $250-500 \mathrm{~mL} / \mathrm{h}$ is appropriate if the corrected serum sodium is normal or elevated; $0.9 \% \mathrm{NaCl}$ at a similar rate is appropriate if corrected serum sodium is low. ${ }^{6}$

Hyperglycemia is typically corrected faster than ketoacidosis. The mean duration of treatment until blood glucose is below $250 \mathrm{mg} / \mathrm{dL}$ and ketoacidosis resolution are 6 and 12 hours, respectively. When the plasma glucose reaches $200 \mathrm{mg} / \mathrm{dL}, 5 \%$ dextrose should be added to replacement fluids to allow continued insulin administration until ketonemia is controlled while at the same time avoiding hypoglycemia. During the recovery phase of DKA, hyperchloremia can develop due to preferential excretion of ketones during rehydration and improved renal perfusion, resulting in a non-anion gap metabolic acidosis. Alternatively, this acidosis may represent a physiological response to resolving DKA rather than a result of the hydration fluid itself.

Using normal saline ( $0.9 \%$ sodium chloride) for DKA management has come under scrutiny. It is proposed that rehydration with normal saline may contribute to hyperchloremia and a hyperchloremic metabolic acidosis with a persisting base deficit leading to prolonged acidosis, associated with renal vasoconstriction, decreased glomerular filtration rate, oliguria, and coagulopathy. The optimal crystalloid solution for DKA management remains unclear. The latest ADA's DKA management guideline was published in July 2009, and currently there is no other updated recommendation regarding this issue.

A recent randomized controlled trial (RCT) in November 2020 compared the clinical effects of balanced crystalloids to saline for the acute treatment of adults with DKA. This RCT study included a subgroup analysis of 172 adults with DKA from two large cluster randomized clinical trials comparing balanced crystalloids and saline. The median time to DKA resolution was 13.0 hours with balanced crystalloids 
and 16.9 hours with saline, with a significant statistical difference. The median (IQR) volume of isotonic fluid administered in the ED and ICU was $4478 \mathrm{~mL}$ (3000-6372 $\mathrm{mL})$. These study results suggest that balanced crystalloids may be preferable to saline for acute management of adults with DKA. ${ }^{7}$

A Cochrane review in 2016 analyzed five small RCTs (total $n=201$ ) comparing subcutaneous injections of insulin analogues (lispro or aspart) to regular insulin IV infusions in patients with mild to moderate DKA. The study revealed that there was no statistical difference in time to resolution of DKA (approximately 11 hours) or rates of hypoglycemia. No deaths occurred. There was no difference in mean length of hospital stays. No serious events associated with diabetic ketoacidosis were observed during insulin lispro treatment. ${ }^{8}$

Another alternative management strategy regarding insulin administration is the initiation of both regular insulin IV infusion and subcutaneous long-acting insulin. A prospective randomized study concluded that once daily subcutaneous insulin glargine administered during intravenous insulin infusions safely prevents rebound hyperglycemia without risk of hypoglycemia. However, this strategy was recommended only in the 2016 NICE guidelines but was not present in the 2009 ADA consensus. Long-acting insulin co-administration with regular insulin IV infusion was well tolerated, associated with faster resolution of acidosis without any adverse effects; patients required a shorter duration of intravenous insulin infusion and had a lower total dose of intravenous insulin and significantly decreased hyperglycemia after discontinuation of the intravenous insulin. This could potentially lead to a shorter ICU (LOS) and reduced costs in the treatment of DKA. ${ }^{9}$ There were no significant differences in outcomes in all studies, except for rebound hyperglycemia, in which patients who received the long-acting subcutaneous glargine had significantly less rebound hyperglycemia at either 12 or 24 hours after regular insulin IV infusion. ${ }^{10}$

\section{REVIEW OF THE DKA ORDER SET AT UNIVERSITY Medical Center}

A retrospective study in 2017 revealed that implementation of a computerized-DKA order set and protocol was associated with improved compliance to the 2009 ADA DKA guidelines, 24-hour fluid resuscitation, initial insulin infusion rate, and time to DKA resolution and appropriate transition to subcutaneous insulin. ${ }^{11}$

A retrospective chart review in 2018 demonstrated an overall improvement in clinical outcomes, times for resolution of DKA, and improvement in LOS in patients who received the order set compared with those who did not receive the order set. Improvements were also noted in arrival to intravenous fluid time, intravenous insulin initiation to discontinuation (resolution) time, arrival to subcutaneous insulin administration time, time from initial to sequential laboratory testing, use of a basal, prandial, and correction insulin approach (physiological mimic), and the frequency of hypoglycemia, while a similar level of glycemic control was achieved. ${ }^{12}$

At University Medical Center in Lubbock, Texas, the medical intensive care unit uses a DKA plan order set. The IV fluids in the DKA order set are normal saline and $5 \%$ dextrose in normal saline; the physician needs to choose the IV flow rate for both IV fluid bolus and maintenance. The physician can initiate regular insulin bolus, for which the default dose is 0.15 units $/ \mathrm{kg}$ IV push 1 time, but there is no recommended initial dose for insulin IV infusion. There are also DKA insulin infusion protocols and hypoglycemia guideline plans. Ondansetron IV, troponin T, creatine kinase, osmolality, and ECG-12 leads are present in the DKA order set.

The criteria for DKA resolution at UMC are different from current ADA guidelines, which are plasma glucose less than $200 \mathrm{mg} / \mathrm{dL}$ and two of the following criteria: plasma bicarbonate greater than or equal to $15 \mathrm{mEq} / \mathrm{L}$, venous $\mathrm{pH}$ greater than 7.3, and anion gap $\leq 12 \mathrm{mEq} / \mathrm{L}$. At UMC, a plasma bicarbonate $\geq 18 \mathrm{mEq} / \mathrm{L}$, a venous $\mathrm{pH}>7.3$, and anion gap $\leq 12 \mathrm{mEq} / \mathrm{L}$ twice consecutively (4 hours apart) are required before bridging to subcutaneous long-acting insulin administration. This time requirement could contribute to a longer time for DKA resolution and longer ICU and hospital stays.

Table 2 shows DKA patient characteristics at UMC; this information was collected during a prospective 
Table 2. Patient Characteristics

\begin{tabular}{|l|l|}
\hline Size, $\mathbf{n}=\mathbf{3 7}$ & Mean \pm SD \\
\hline Age, years & $38.1 \pm 18.5$ \\
\hline Gender, Male, n (\%) & $19(51.4)$ \\
\hline APACHE-II Score & $10.2 \pm 4.2$ \\
\hline $\begin{array}{l}\text { Precipitating Cause } \\
\text { of DKA, n (\%) }\end{array}$ & $\begin{array}{l}\text { 1. Poor compliance }=16(43.2) \\
\text { 2. Infection }=11(29.7) \\
\text { 3. Pump Malfunction }=3(8.15) \\
\text { 4. Newly diagnosed DM }=2(5.4) \\
\text { 5. Substance abuse }=2(5.4) \\
\text { 6. Corticosteroid use }=1(2.7) \\
\text { 6. Unknown }=1(2.7)\end{array}$ \\
\hline $\begin{array}{l}\text { Initial Laboratory } \\
\text { Values }\end{array}$ & Mean \pm SD \\
\hline Blood glucose, mg/dL & $546.4 \pm 296.3$ \\
\hline Anion gap, mEq/L & $31.8 \pm 7.8$ \\
\hline Total CO2, mEq/L & $10.1 \pm 5.5$ \\
\hline pH & $7.2 \pm 0.2$ \\
\hline Creatinine, mg/dL & $1.1 \pm 1$ \\
\hline BUN, mg/dL & $29.7 \pm 29.3$ \\
\hline Albumin, gm/dL & $4.4 \pm 0.5$ \\
\hline
\end{tabular}

APACHE-II score-Acute Physiologic Assessment and Chronic Health Evaluation Score; DKA-diabetic ketoacidosis; $\mathrm{CO} 2$-total bicarbonate in chemistry panel; BUN-blood urea nitrogen.

study measuring advanced glycation end-products in patients with DKA and hyperglycemia. ${ }^{13}$ The mean age was $38.1 \pm 18.5$ years; $19 \%$ were male. Most of the precipitating causes were poor compliance, infection, and insulin pump malfunction. Mean blood sugar, anion gap, bicarbonate, and $\mathrm{pH}$ at the time of diagnosis were $546.4 \pm 296.3 \mathrm{mg} / \mathrm{dL}, 31.8 \pm 7.8 \mathrm{mEq} / \mathrm{L}$, $10.1 \pm 5.5 \mathrm{mEq} / \mathrm{L}$, and $7.2 \pm 0.2$, respectively.

Table 3 reports the time until AG closed twice was $19.2 \pm 12.8$ hours, and total IV fluid until AG closed was $3694.9 \pm 2484.6 \mathrm{~mL}$. Table 4 reports the number of BMP and $A B G$ in the first 24 hours were $12.2 \pm 13.7$, and $1.8 \pm 1.6$, respectively. The length of ICU-stays and hospital stays were $2.5 \pm 3.8$ days and $4.8 \pm 7.4$ days, respectively, as shown in Table 5.
Table 3. Diabetic Ketoacidosis Outcome: Management Characteristics

\begin{tabular}{|l|l|}
\hline Number of Patients $(\mathbf{n})$ & Mean \pm SD \\
\hline $\begin{array}{l}\text { Time until AG closed twice, hours, } \\
\mathrm{n}=37\end{array}$ & $19.2 \pm 12.8$ \\
\hline $\begin{array}{l}\text { Total IV fluid until AG closed, } \mathrm{mL}, \\
\mathrm{n}=37\end{array}$ & $3694.9 \pm 2484.6$ \\
\hline $\begin{array}{l}\text { Total IV insulin in } 1^{\text {st }} 24 \text { hours, units, } \\
\mathrm{n}=37\end{array}$ & $69.5 \pm 49.9$ \\
\hline $\begin{array}{l}\text { Total IV insulin in } 2^{\text {nd }} \text { hours, units, } \\
\mathrm{n}=19\end{array}$ & $45.2 \pm 43.2$ \\
\hline $\begin{array}{l}\text { Total IV insulin in } 4^{\text {th }} \text { hours, units, } \\
\mathrm{n}=4\end{array}$ & $40.7 \pm 26.8$ \\
\hline $\begin{array}{l}\text { Fluid balance in } 1^{\text {st }} 24 \text { hours, } \mathrm{mL}, \\
\mathrm{n}=37\end{array}$ & $4108.3 \pm 2048.5$ \\
\hline $\begin{array}{l}\text { Fluid balance in } 2^{\text {nd }} 24 \text { hours, } \mathrm{mL}, \\
\mathrm{n}=37\end{array}$ & $1158.5 \pm 1816$ \\
\hline $\begin{array}{l}\text { Fluid balance in } 4^{\text {th }} 24 \text { hours, } \mathrm{mL}, \\
\mathrm{n}=16\end{array}$ & $241.0 \pm 1060$ \\
\hline $\begin{array}{l}\text { Maximum BG on } 1^{\text {st }} 24 \text { hours, } \mathrm{mg} / \mathrm{dL}, \\
\mathrm{n}=37\end{array}$ & $551.4 \pm 320.9$ \\
\hline $\begin{array}{l}\text { Maximum BG on } 2^{\text {nd }} 24 \text { hours, } \mathrm{mg} / \mathrm{dL}, \\
\mathrm{n}=37\end{array}$ & $303.4 \pm 79.3$ \\
\hline $\begin{array}{l}\text { Maximum BG on in } 4^{\text {th }} 24 \text { hours, } \\
\text { mg/dL, } \mathrm{n}=17\end{array}$ & $277.5 \pm 89.7$ \\
\hline $\begin{array}{l}\text { Minimum BG on } 1^{\text {st }} 24 \text { hours, } \mathrm{mg} / \mathrm{dL}, \\
\mathrm{n}=37\end{array}$ & $116.5 \pm 35.6$ \\
\hline $\begin{array}{l}\text { Minimum } \mathrm{BG} \text { on } 2^{\text {nd }} 24 \text { hours, } \\
\mathrm{mg} / \mathrm{dL}, \mathrm{n}=37\end{array}$ & $120.1 \pm 62.8$ \\
\hline $\begin{array}{l}\text { Minimum } \mathrm{BG} \text { on in } 4^{\text {th }} 24 \text { hours, } \\
\mathrm{mg} / \mathrm{dL}, \mathrm{n}=17\end{array}$ & $132.5 \pm 57.0$ \\
\hline
\end{tabular}

AG-anion gap; IV-intravenous; BG-blood glucose level.

\section{Proposal to improve our DKA management}

Patients enrolled in this prospective study had the typical laboratory test results expected in diabetic ketoacidosis. The time to anion gap closure twice was approximately 19 hours. The fluid and insulin required during this initial period were $3700 \mathrm{~mL}$ of fluid and 70 units of regular insulin. Based on our literature review, we suggest that DKA management could be 
Table 4. Diabetic Ketoacidosis Outcome: Number of Laboratory Tests

\begin{tabular}{|l|c|}
\hline Number of Laboratory Tests & Mean \pm SD \\
\hline Number of BMP & $12.2 \pm 13.7$ \\
\hline Number of ABG $1^{\text {st }} 24$ hours & $1.8 \pm 1.6$ \\
\hline Number of POC BG in $1^{\text {st }} 24$ hours & $21.2 \pm 6$ \\
\hline Number of POC BG in $2^{\text {nd }} 24$ hours & $11.3 \pm 6.6$ \\
\hline Number of POC BG in $4^{\text {th }} 24^{*}$ hours & $7.8 \pm 5.6$ \\
\hline
\end{tabular}

BMP-basic metabolic panel; ABG-arterial blood gas; POC-point of care; BGblood glucose level; ICU-intensive care unit; * Total number of patients who had POC blood glucose in $4^{\text {th }} 24$ hours was 17 patients.

improved in our hospital by following ADA guidelines for DKA resolution criteria, which are plasma glucose less than $200 \mathrm{mg} / \mathrm{dL}$ and 2 of the following criteria: plasma bicarbonate $\geq 15 \mathrm{mEq} / \mathrm{L}$, venous $\mathrm{pH}$ greater than 7.3 , and anion gap $\leq 12 \mathrm{mEq} / \mathrm{L}$. This should decrease the length of ICU-stays and hospital stays. A subcutaneous insulin protocol for mild DKA could decrease ICU admissions and the cost of hospitalization. The DKA order set should be updated, saline should be replaced with balanced crystalloids, and there should be the option to start daily long-acting insulin to prevent rebound hyperglycemia. Finally, the number of lab tests used during management should be reviewed and possibly reduced. This information in

Table 5. Complications and Length of Stay

\begin{tabular}{|l|c|}
\hline Complication and Length of Stay & n (37) \\
\hline Acute kidney injury & 7 \\
\hline Mechanical ventilation & 4 \\
\hline GI bleeding & 2 \\
\hline Pancreatitis & 1 \\
\hline Thrombocytopenia & 1 \\
\hline Vasopressors use & 0 \\
\hline Hospital acquired infection & 0 \\
\hline ICU length of stay, days** & $2.5 \pm 3.8$ \\
\hline Hospital length of stay, days** & $4.8 \pm 7.4$ \\
\hline Mortality & 0 \\
\hline
\end{tabular}

GI-gastrointestinal bleeding; ICU-intensive care unit; ** mean \pm SD this article provides background information for comparisons with future changes in DKA protocols.

Article citation: Motes A, Fabiana Fernandez F, Edriss $\mathrm{H}$. Analysis of a DKA protocol: Laboratory tests and outcomes. The Southwest Respiratory and Critical Care Chronicles 2021;9(40):20-26

From: Department of Internal Medicine (AM, FF), Texas Tech University Health Sciences Center, Lubbock, Texas; Pulmonary and Critical Care Medicine (HE), CHI Saint Joseph Hospital, Lexington, Kentucky

Submitted: $6 / 14 / 2021$

Accepted: 6/27/2021

Reviewer: Shaili Felton MD

Conflicts of interest: none

This work is licensed under a Creative Commons Attribution-ShareAlike 4.0 International License.

\section{REFERENCES}

1. National Diabetes Statistics Report, 2020 | CDC. Accessed 04 April 2021.

2. Wilson C, Krakoff J, Gohdes D. Ketoacidosis in Apache Indians with non-insulin-dependent diabetes mellitus. Arch Intern Med 1997;157:2098-100.

3. Wolfsdorf J, Craig ME, Daneman D, et al. Diabetic ketoacidosis in children and adolescents with diabetes. Pediatr Diabetes 2009;10(suppl 12):118-133.

4. Bohn D, Daneman D. Diabetic ketoacidosis and cerebral edema. Curr Opin Pediatr 2002 Jun;14(3):287-91.

5. Tran TTT, Pease A, Wood AJ, et al. Review of evidence for adult diabetic ketoacidosis management protocols. Front Endocrinol 13 June 2017. https://doi.org/10.3389/fendo.2017.00106.

6. Abbas E. Kitabchi, Guillermo E. et al. Hyperglycemic crises in adult patients with diabetes. Diabetes Care Jul 2009; 32(7):1335-1343.

7. Self WH, Evans CS, Jenkins CA, et al. Clinical effects of balanced crystalloids vs saline in adults with diabetic ketoacidosis: a subgroup analysis of cluster randomized clinical trials. JAMA Netw Open 2020;3(11):e2024596. doi:10.1001/ jamanetworkopen.2020.24596.

8. Andrade Castellanos CA, Colunga Lozano LE, Delgado Figueroa N, et al. Subcutaneous rapid acting insulin analogues for diabetic ketoacidosis. Cochrane Database of Systematic Reviews 2016, Issue 1. Art. No.: CD011281. DOI: 10.1002/ 14651858.CD011281.pub2. Accessed 01 June 2021. 
9. Barski L, Brandstaetter E, Sagy I, et al. Basal insulin for the management of diabetic ketoacidosis. Eur J Intern Med 2018 Jan; 47:14-16.

10. Bunn S, Margo Halm M. Long-acting insulin on the road to recovery with diabetic ketoacidosis. Am J Crit Care 2016;25 (3):277-280.

11. Lalibertea B, Yeung SYA, Gonzales JP. Impact of diabetic ketoacidosis management in the medical intensive care unit after order set implementation. International J Pharmacy Practice 2017;25:238-243.

12. Blair AMJ, Hamilton BK, Spurlock A. Evaluating an order set for improvement of quality outcomes in diabetic ketoacidosis. Advanced Emergency Nursing J 2018;40(1):59-72.

13. Edriss H, Molehin AJ, Selvan K, et al. Advanced glycation end products and glycosaminoglycans in patients with diabetic ketoacidosis. J Investig Med 2020 Mar;68(3):738-74. 